\title{
Erratum to: Neuroimmune Pharmacology from a Neuroscience Perspective
}

\author{
Nicole A. Northrop • Bryan K. Yamamoto
}

Published online: 14 September 2010

(C) Springer Science+Business Media, LLC 2010

Erratum to: J Neuroimmune Pharmacol

DOI 10.1007/s11481-010-9239-2

The last name of the first author should be given as Nicole A. Northrop, not Nicole A. Northrup, as originally published. The correct name is also shown above.

The online version of the original article can be found at http://dx.doi. org/10.1007/s11481-010-9239-2.

\footnotetext{
N. A. Northrop

Department of Pharmacology and Experimental Therapeutics,

Boston University School of Medicine,

Boston, MA 02118, USA

B. K. Yamamoto $(\square)$

Department of Neurosciences,

University of Toledo College of Medicine,

3000 Arlington Avenue,

Toledo, OH 43614, USA

e-mail: Bryan.Yamamoto@utoledo.edu
} 